\title{
Mentoria virtual para estudantes de medicina em tempos de covid-19
}

\author{
Virtual mentoring for medical students in the Covid-19 times
}

\author{
Izabel Cristina Rios ${ }^{1}$ \\ Martim Elviro de Medeiros Junior ${ }^{1}$ \\ izabel.rios@santamarcelina.edu.br \\ Maria Teresa de Almeida Fernandes ${ }^{1}$ \\ martim.medeiros@santamarcelina.edu.br \\ Edson Vanderlei Zombini \\ teresa.fernandes@santamarcelina.edu.br \\ Maira Kassabian Oliveira Pacheco' ${ }^{1}$ \\ edson.zombini@santamarcelina.edu.br \\ Elcio Gomes Mascarenhas ${ }^{1}$ \\ Joselita Batista Azuma ${ }^{1}$ \\ maira.pacheco@santamarcelina.edu.br \\ Pedro Felix Vital Junior ${ }^{1}$ \\ elcio.mascarenhas@santamarcelina.edu.br \\ joselita.azuma@santamarcelina.edu.br \\ pedro.vital@santamarcelina.edu.br
}

\section{RESUMO}

Introdução: Durante a pandemia de Sars-CoV-2, as escolas adotaram distanciamento social e atividades a distância que, segundo estudos recentes, sugerem o aumento de depressão, ansiedade e distúrbios de comportamento entre estudantes universitários. A formação médica que é marcada por forte estresse psicológico contou com esse agravante, reforçando a necessidade de ações de cuidado destinadas aos alunos, como os programas de mentoria. A mentoria de alunos oferece suporte empático e desenvolvimental que estimula autocuidado, bem-estar e resiliência.

Relato de experiência: Um grupo de professores-mentores de uma escola médica adaptou atividades de mentoria para um modelo remoto dirigido aos alunos veteranos que já participavam da mentoria e separadamente aos estudantes recém-ingressos no curso médico. No formato remoto, manteve-se a proposta de mentoria como "lugar de conversa", mas em plataforma digital. O acompanhamento técnico da proposta se deu por meio de encontros remotos do grupo de mentores e de consulta aos alunos por meio de questionário autoaplicado on-line. Os dados obtidos foram submetidos à análise de conteúdo.

Discussão: De março a dezembro de 2020, realizaram-se 109 encontros de mentoria virtual. Os alunos consideraram satisfatórios os encontros remotos em termos de qualidade das discussões, atuação do mentor e ambiente emocional. A desenvoltura interativa variou entre grupos, mas foi facilitada nos grupos de veteranos. Entre os alunos ingressantes, o tema recorrente esteve atrelado ao sentimento de medo: de ter mau desempenho nas provas, de perder o semestre e de não aprender. Entre os veteranos, destacaram-se as dificuldades de adaptação ao ensino a distância e de organização diante do excesso de atividades, e a diminuição das atividades práticas. Todos os grupos relataram medo da pandemia, da morte e de a situação financeira dos pais piorar, além de tristeza em razão de perda de parentes pela Covid-19. Chamou a atenção dos mentores o fato de os alunos, mesmo em um espaço acolhedor, manterem fechadas as câmeras.

Conclusão: Para os mentores e discentes, a mentoria virtual funcionou como importante suporte ao aluno. Veteranos e calouros relataram que se sentiam cuidados, fortalecidos e gratos, sugerindo que os encontros permitiram uma boa interação das pessoas e a produção de efeitos benéficos perceptíveis. Um limite deste estudo foi o tempo de experiência. Assim sendo, recomenda-se manter tal investigação.

Palavras-chave: Pandemias; Infecção por Coronavírus; Mentoria; Educação a Distância; Educação Médica.

\section{ABSTRACT}

Introduction: Throughout the SARS-COV-2 pandemic, schools had to adopt social distancing and remote learning, which, according to recent studies suggest an increase in depression, anxiety and behavioral disorders among university students. Medical training, which has a heavy load of psychological issues had to face this aggravating factor, reinforcing the need for support actions for students, such as mentoring programs. Mentoring programs offer empathetic and developmental support that encourages self-care, well-being and resilience.

Experience report: A group of teacher-mentors from a medical school adapted the mentoring activities to a remote model, offering them to upperlevel students who had already participated in mentoring, and, separately, to students who were newly enrolled in the medical course. In the remote format, the mentoring proposal was maintained as a "place of conversation", but on a digital platform. The technical follow-up was carried out by remote meetings of the group of teacher-mentors, and consultation with students through a self-administered online questionnaire. The data obtained were submitted to content analysis.

Discussion: From March to December, 109 virtual mentoring meetings were held. The students considered the meetings satisfactory in terms of the quality of discussions, the mentors' attitudes and the emotional environment. The interactive development varied among groups, but it was observed to be easier in the groups of upper-level students. Among the newly-enrolled students, the recurring topic was the fear of poor performance in the tests, losing the semester, or not learning. Upper-level students highlighted the difficulties of adapting to remote learning and organizing their activities, and the decrease in practical activities. All groups reported fear of the pandemic, of death, of the worsening of parents' financial situation, and sadness about the loss of relatives to COVID-19. It drew the attention of the mentors the fact that the students, even in a welcoming space, kept the cameras turned off.

Conclusion: For mentors and students, virtual mentoring worked as an important student support system. Upper-level students and first-year ones reported feeling cared for, supported and grateful, suggesting that the meetings allowed a good interaction and produced beneficial effects. A limitation of this study was the duration of the experiment. Therefore, it is recommended that the research be maintained.

Keywords: Pandemics; Coronavirus Infection; Mentoring, Remote Education; Medical Schools.

1 Faculdade Santa Marcelina, São Paulo, São Paulo, Brasil. 


\section{INTRODUÇÃO}

Em 2020, devido à pandemia do severe acute respiratory syndrome coronavirus (Sars-CoV-2), as escolas fecharam suas portas por razões sanitárias ${ }^{1}$. Pouco tempo depois da suspensão das aulas, várias delas adaptaram o ensino para a modalidade remota. De um momento para o outro, o ambiente educacional sofreu uma mudança drástica, colocando para professores e alunos o desafio de ensinar e aprender em cenário de distanciamento social. Essa mudança de rumo se deu em meio a dúvidas, incertezas e angústias de todos os lados.

Nas escolas médicas, essa situação tornou-se mais preocupante, visto que a formação médica, por sua natureza social e acadêmica, é normalmente fonte de estresse para os estudantes ${ }^{2}$. Estudos de revisão ${ }^{3,4}$ da literatura e uma investigação de abrangência nacional realizada nos Estados Unidos ${ }^{5}$ mostraram que a prevalência de estresse nos estudantes de Medicina era significativamente maior que da população em geral, sendo os alunos mais jovens os mais acometidos. No Brasil, um estudo de revisão sistemática ${ }^{6}$ realizado em 2017 mostrou que cerca de 50\% dos estudantes de Medicina referiam estresse psicológico. Quando não há recursos de enfrentamento, o estresse psicológico provoca decepção com a escolha profissional, sofrimento mental e compromete o desempenho escolar.

A preocupação com os estudantes de Medicina também se justificou a partir de estudos sobre as implicações da pandemia na saúde mental deles ${ }^{7-9}$. $O$ isolamento social decorrente da pandemia de Sars-CoV-2, assim como as mudanças do ensino presencial para remoto e a distância, aumentou a prevalência de quadros de depressão, ansiedade e distúrbios de comportamento entre estudantes universitários. Esses fatos reforçaram a necessidade de a escola médica ofertar aos alunos algum tipo de cuidado.

Entre as ofertas possíveis, destacam-se os programas de mentoria com a participação de professores que, com sua experiência de vida e de profissão, conseguem ajudar o aluno a percorrer o caminho acadêmico com um pouco mais de segurança emocional. Revisões da literatura médica ${ }^{10,11}$ identificam a mentoria como uma prática de oferta de suporte empático e desenvolvimental para autocuidado, bem-estar e resiliência. Os resultados apresentados nesses estudos $^{10,11}$ mostraram melhora da qualidade de vida do estudante e do desenvolvimento de competências relacionais, ainda que com a ressalva de que os programas de mentoria precisariam aprimorar seus métodos de avaliação com vistas à produção de evidências de impacto mais robustas. No Brasil, os estudos também mostraram bons resultados dos programas de mentoria na formação médica ${ }^{12,13}$. A mentoria de alunos é, portanto, uma estratégia de desenvolvimento de competências para o enfrentamento das dificuldades inerentes à formação médica, que fortalece a construção da identidade profissional do futuro médico, respeitando suas características e necessidades pessoais.

Diante dessas considerações, em meio à pandemia de Sars-CoV-2 e ao forte impacto percebido na educação médica e na relação professor-aluno determinada pelo distanciamento social, um grupo de professores-mentores de um programa de mentoria já institucionalizado há dois anos adaptou suas atividades de mentoria presencial para um modelo remoto dirigido aos alunos que já participavam da mentoria e separadamente aos estudantes do primeiro período do curso médico. Para os veteranos, o objetivo foi dar continuidade ao programa e aos benefícios já propiciados. Para os calouros, o objetivo foi oferecer acolhimento e sustentação para lidar com as mudanças de vida representadas pelo ingresso na Faculdade de Medicina em situação de isolamento. Essa experiência de mentoria remota é o objeto de relato e análise neste artigo.

\section{RELATO DA EXPERIÊNCIA}

A escola médica em que se deu a experiência é uma instituição nova cujo padrão de ensino foi avaliado pelo Ministério da Educação (MEC) com nota 5 (nota máxima). Trata-se de uma instituição de natureza privada, localizada na cidade de São Paulo, que tem como uma de suas principais características, segundo seus alunos, ser um lugar acolhedor em que, de modo geral, a relação professor-aluno é boa e próxima. Esse aspecto é bastante importante no ensino médico, em que o professor é modelo para a formação humanística basilar da prática médica ${ }^{14}$.

A exemplo dessa proximidade, o próprio programa de mentoria, criado em 2018, foi cuidadosamente elaborado com os alunos. Mediante a realização de várias rodas de conversa, os alunos puderam escolher o modelo de mentoria mais adequado às suas expectativas e necessidades, assim como ajudar a escolher os professores-mentores. Doze professores foram escolhidos por critérios qualitativos definidos por: ter compromisso com o ensino médico, ser empático e se comunicar bem, ser reconhecido pelos alunos como um bom professor e ter interesse e disponibilidade para ser mentor. Realizou-se um curso de preparação de mentores, no qual se utilizaram metodologias ativas, tendo em vista o desenvolvimento das seguintes competências para a atuação do mentor: 1. praticar uma escuta atenta, 2. coordenar uma roda de conversa, 3. estimular pensamento crítico, 4. identificar bias da construção do pensamento na formação médica e 5 . promover comunicação efetiva e empática. O modelo final do programa de mentoria se deu com a participação dos próprios mentores na sua estruturação. 
O programa assim construído adotou caráter de extensão cultural, contando como atividade complementar que acumula créditos, ofertado semestralmente aos alunos interessados.

\section{Mentoria para alunos veteranos}

Participaram dessa mentoria dez grupos de alunos veteranos de diversos períodos letivos, que ingressaram nos grupos por livre escolha e afinidade com o mentor.

Antes da pandemia, os encontros de mentoria ocorriam presencialmente no formato de rodas de conversa mensais, em horário e local programados pela coordenação do programa. Os temas de conversa eram livres, trazidos pelos alunos e tratados pelo grupo de forma acolhedora e reflexiva, buscando ampliar a compreensão dos temas e, se pertinente, elaborar respostas coletivas aos problemas.

Nesse modelo, não cabe ao mentor dar respostas baseadas nas suas opiniões, mas estimular o grupo a pensar e encontrar formas de lidar com o cotidiano de ser estudante de Medicina e futuramente médico, de modo a fortalecer os alunos para o enfrentamento de suas dificuldades. Entretanto, em muitos momentos, é importante que o mentor recorra às suas próprias experiências, memórias e histórias de vida para proporcionar aos alunos um depoimento vivo de significados biográficos.

\section{Mentoria para alunos ingressantes}

Em atenção aos alunos ingressantes no curso médico, tidos como potencialmente mais propensos à desorientação e ao estresse, e que, após duas semanas de ingresso, tiveram que se adaptar a um ambiente desconhecido e ao ensino remoto, criaram-se nove grupos de "calouros" no primeiro semestre e oito no segundo semestre, com uma agenda de encontros mensais voltados especificamente para eles. Os grupos foram formados aleatoriamente, pois os alunos pouco conheciam os professores, os colegas e mesmo o programa de mentoria. Os temas continuaram livres, mas, com base em experiências anteriores de assistência psicopedagógica a alunos ingressantes, elegeram-se assuntos que poderiam ser abordados pelos mentores caso lhes parecessem adequados ao grupo, como: escolha da medicina, mudanças de vida de estudante de ensino médio para estudante de faculdade e como se organizar para estudar.

\section{Adaptações para a mentoria virtual}

A adaptação para o formato remoto manteve a proposta de ser "lugar de conversa", mas, para facilitar o encontro, optouse por utilizar horário previsto na grade curricular ou conforme conveniência de alunos e mentores. Optou-se pela plataforma Zoom por ser uma ferramenta institucional para atividades presenciais remotas.

Como antes da pandemia, o acompanhamento técnico do programa de mentoria se deu por meio de encontros do grupo de mentores com a coordenação do programa e de consulta aos alunos por meio de questionário on-line autoaplicado, com perguntas fechadas e respostas em escala Likert e perguntas abertas para respostas discursivas. Os encontros de acompanhamento ocorreram em modo remoto. Neles, realizou-se o registro escrito das opiniões e percepções dos mentores apresentadas na forma de narrativas livres sobre o funcionamento dos grupos e os assuntos trazidos pelos alunos.

Os dados obtidos dos questionários respondidos pelos alunos e dos registros dos encontros com os mentores foram submetidos à análise de conteúdo e triangulados para dar complementaridade aos resultados de avaliação e monitoramento do programa.

\section{Resultados da experiência \\ Análise descritiva}

Nos meses de março a dezembro de 2020, foram realizados 109 encontros de mentoria virtual e 10 encontros de monitoramento entre mentores e coordenação, e 110 alunos participantes do programa responderam aos questionários de avaliação.

A participação numérica dos alunos do primeiro período (ingressantes) no primeiro e segundo semestres de 2020 e dos demais alunos (veteranos) na mentoria pode ser observada no quadro 1.

Quadro 1. Números absolutos e relativos de alunos ingressantes e veteranos que participaram da mentoria virtual no período de março a dezembro de 2020.

\begin{tabular}{|c|c|c|c|c|c|c|}
\hline \multirow[t]{3}{*}{ Tempo no curso médico } & \multicolumn{2}{|c|}{ Matriculados } & \multicolumn{2}{|c|}{ Mentoria } & \multicolumn{2}{|c|}{ Participação } \\
\hline & \multicolumn{2}{|c|}{ No } & \multicolumn{2}{|c|}{ No } & \multicolumn{2}{|c|}{$\%$} \\
\hline & $\begin{array}{l}\text { Primeiro } \\
\text { semestre }\end{array}$ & $\begin{array}{l}\text { Segundo } \\
\text { semestre }\end{array}$ & $\begin{array}{l}\text { Primeiro } \\
\text { semestre }\end{array}$ & $\begin{array}{l}\text { Segundo } \\
\text { semestre }\end{array}$ & $\begin{array}{l}\text { Primeiro } \\
\text { semestre }\end{array}$ & $\begin{array}{l}\text { Segundo } \\
\text { semestre }\end{array}$ \\
\hline Ingressantes & 82 & 108 & 58 & 89 & 70 & 83 \\
\hline Veteranos & & 720 & & 95 & & 13 \\
\hline Total & & 910 & & 242 & & 27 \\
\hline
\end{tabular}


O número de participantes por encontro variou de um a dez alunos, conforme o grupo, mantendo média de cinco discentes por grupo. Nos dez grupos de alunos veteranos, a participação se deu no mesmo padrão dos encontros presenciais. Em alguns grupos, os mentores observaram aumento do número de participantes nos encontros virtuais comparativamente aos encontros presenciais.

No quadro 2, apresenta-se a satisfação dos alunos relativamente aos itens qualidade das discussões e reflexões, atuação do mentor e acolhimento, ambiente e apoio emocional, segundo as respostas deles nos questionários de avaliação do programa.

Solicitados a atribuir uma nota de 0 a 10 aos encontros remotos dos seus grupos, a mentoria virtual obteve nota média de 9,36.

Quanto à preferência pelas modalidades, 22\% dos alunos mencionaram o modo presencial; 25\%, o remoto; e a maioria (53\%), o modelo híbrido (presencial e remoto).

Em que pese a boa avaliação da mentoria virtual, quando comparadas as respostas dos alunos veteranos em 2019 com

Quadro 2. Porcentagem de respostas "muito bom" e "bom" em uma escala Likert (muito ruim, ruim, bom e muito bom) para itens de satisfação dos alunos com a mentoria virtual, no período de março a dezembro de $2020-\mathrm{N}=110$.

\begin{tabular}{ccc}
\hline Elemento analisado & Muito bom & Bom \\
\hline Discussões e reflexões & 70,0 & 29,1 \\
Atuação do mentor & 93,6 & 5,5 \\
Ambiente e apoio emocional & 81,8 & 18,2 \\
\hline
\end{tabular}

2020, observou-se uma pequena diferença a favor do modo presencial, como mostra o Gráfico 1.

\section{Análise de conteúdo}

Os mentores relataram que os encontros remotos foram bem-aceitos pelos alunos, percepção confirmada pelos discentes nos questionários. De modo geral, os alunos se sentiram à vontade para falar, dar opiniões e discutir assuntos. Essa desenvoltura interativa variou entre os grupos, mas foi facilitada nos grupos de alunos e mentores que já se conheciam previamente. Os grupos de alunos ingressantes do primeiro semestre exigiram dos mentores uma condução mais ativa para facilitar a interação, entretanto o grupo de ingressantes do segundo semestre mostrou-se bastante à vontade e participou ativamente nos encontros.

Chamou a atenção dos mentores a dificuldade de alguns alunos abrirem a câmera, fato que tem sido recorrentemente observado no ensino remoto. Mesmo no encontro de mentoria, que é por definição um espaço mais "protegido", especialmente no primeiro semestre do ano, foi difícil fazer com que os alunos aceitassem expor a própria imagem. No segundo semestre, observou-se maior número de alunos com a câmera aberta. Em alguns grupos, ficou evidente que a abertura das câmeras se deu no momento em que os alunos perceberam que o espaço da mentoria continuava "protegido" também no ambiente remoto. Em outros grupos, os alunos abriram as câmeras quando sentiram confiança em expor parte de sua vida mais pessoal e reservada ao ambiente doméstico.

$\mathrm{Na}$ análise de conteúdo sobre dados narrativos de mentores e discursivos de discentes - considerando-se 17 grupos de ingressantes e separadamente dez grupos de veteranos -, aglutinaram-se os conteúdos recorrentes nos

Gráfico 1. Porcentagem de respostas "muito bom" em uma escala Likert (muito ruim, ruim, bom e muito bom) para acolhimento, reflexões e participação dos alunos nos encontros de mentoria, segundo veteranos em 2019 e 2020 - N = 53.

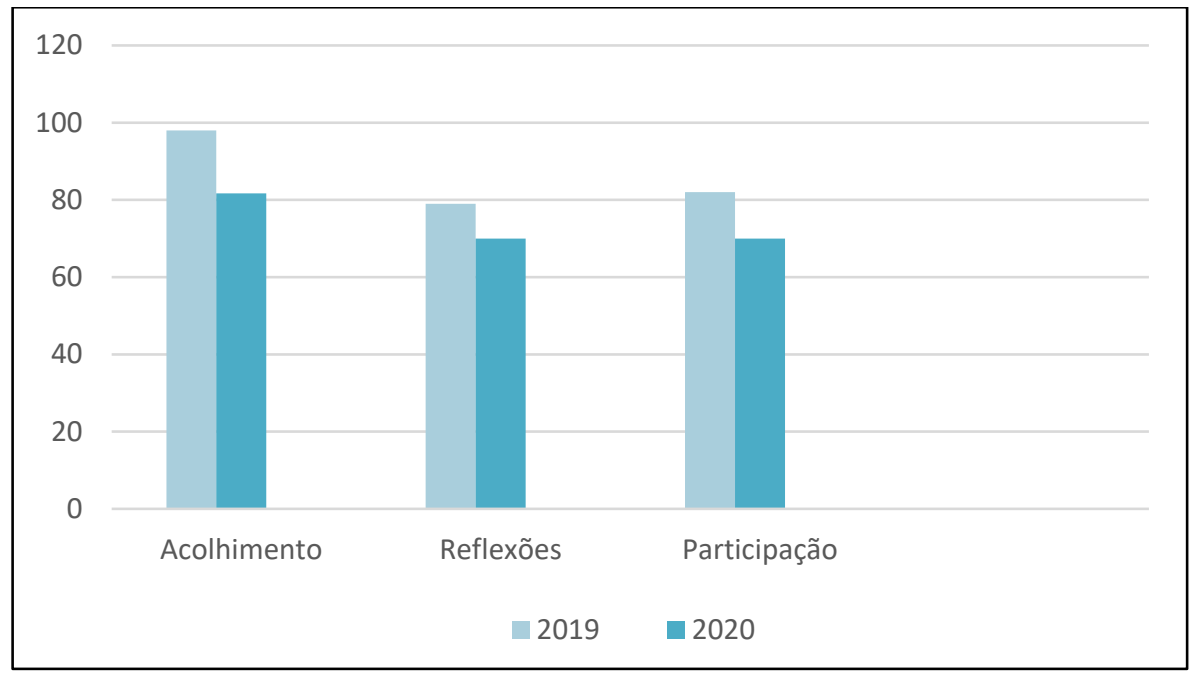


encontros em duas categorias analíticas: 1. preocupações com a vida acadêmica e 2. preocupações com a sua vida e a dos outros, conforme disposto nos quadros 3 e 4.

Os conteúdos relativos ao excesso de atividades das disciplinas, à preocupação com a família e ao medo da pandemia foram comuns entre ingressantes e veteranos. Os ingressantes referiram mais dificuldades na gestão do tempo de estudar e dúvidas sobre o cotidiano da faculdade, enquanto os veteranos mostraram-se mais preocupados com a falta de aulas práticas, o internato e o quanto conseguiriam se preparar para as provas de residência.

Para mentores e alunos, a mentoria virtual foi um importante suporte ao aluno perante o distanciamento social decorrente à pandemia.

Para os ingressantes, os encontros foram bons para compartilhar ideias, histórias, expectativas e medos. Os encontros também propiciaram o efeito de aproximá-los dos colegas e da faculdade, tida como ainda "muito distante"

Quadro 3. Categorias analíticas relativas aos conteúdos recorrentes nos encontros de mentoria virtual para alunos ingressantes no período de março a dezembro de 2020.

\section{Preocupações com a vida acadêmica}

- Excesso de atividades a distância e dificuldades em organizar o tempo para estudar.

- Medo do desconhecido acadêmico, especialmente das provas e exigências de desempenho; e consequentemente medo de perder o semestre por não conseguir estudar o suficiente e não aprender tudo o que precisa.

- Dúvidas sobre se de fato é possível aprender medicina no ensino a distância

\section{Preocupações com a sua vida e a dos outros}

- Necessidade de muitas adaptações na vida familiar para "encaixar" a vida acadêmica na rotina de casa.

- Preocupações com a situação financeira da família (pais autônomos sem renda ou pais com vínculos empregatícios, mas com redução salarial).

- Dificuldades relacionais com os pais que, trabalhando em home office, extrapolam o tempo de trabalho, tendo pouco tempo para dar-lhes atenção.

- Medo de que os pais adoeçam, luto e tristeza pelos parentes doentes ou que morreram de coronavirus disease 2019 (Covid-19).

- A vida na pandemia, o cansaço de ficar em casa, e outras coisas que gostariam de fazer além da medicina.

- Desejo do retorno à vida fora de casa, à faculdade, às aulas, às festas, mas medo de se contaminar e levar a contaminação para dentro de casa.

Quadro 4. Categorias analíticas relativas aos conteúdos recorrentes nos encontros de mentoria virtual para alunos veteranos no período de março a dezembro de 2020.

\section{Preocupações com a vida acadêmica}

- Dificuldades de adaptação ao ensino A distância, mas ganho de competências no uso das tecnológicas de comunicação.

- Excesso de atividades em várias disciplinas, dificuldade para se organizar no tempo, cansaço, estresse e exaustão decorrentes desse excesso.

- Dificuldade de estudar em casa, pois os familiares os requisitam mais para ajudar nas atividades domésticas.

- Preocupações com a diminuição das aulas práticas e seu impacto na aprendizagem.

- Angústias por não saberem como será o internato em meio à pandemia, se conseguirão se preparar para as provas de residência e como será o futuro profissional.

\section{Preocupações com a sua vida e a dos outros}

- Necessidade de muitas adaptações na vida familiar para "encaixar" a vida acadêmica na rotina de casa.

- Preocupações com a situação financeira da família (pais autônomos sem renda ou pais com vínculos empregatícios, mas com redução salarial).

- Medo de que os pais adoeçam, luto e tristeza pelos parentes doentes ou que morreram de Covid-19.

- Medo da pandemia, da doença, da morte e de como será o futuro pós-pandemia.

- Angústias decorrentes das dúvidas sobre até quando vai durar a pandemia.

- Desejo do retorno à vida fora de casa, à faculdade, às aulas, às festas, mas medo de se contaminar e levar a contaminação para dentro de casa. 
de sua realidade. Os calouros afirmaram que os encontros permitiram a troca de experiências que poderiam ser muito angustiantes se vivenciadas de forma solitária.

No caso dos veteranos, os encontros diminuíram a sensação de estarem perdidos e os ajudaram a lidar com as próprias angústias, como não se sentirem sozinhos com suas dúvidas e seus problemas. Esses discentes reforçaram a importância da mentoria para aproximar alunos e faculdade, além de facilitar a troca de experiências e a solução de problemas do cotidiano acadêmico.

\section{REFLEXÕES SOBRE A EXPERIÊNCIA}

A mentoria como programa institucional ${ }^{15}$ sinaliza o compromisso da escola médica com a boa formação acadêmica conjugada ao fortalecimento emocional do aluno. Ao promover a mentoria, a escola médica cultiva a aproximação entre as pessoas, as relações de pertencimento, a ajuda mútua e a cultura institucional de desenvolvimento humano.

Os encontros de mentoria se caracterizam como espaços de produção de intersubjetividade ${ }^{11,15}$, posto que os participantes estabelecem relações de compreensão das diferentes histórias de vida, valores e expectativas de ser médico. Essa construção se dá por meio de comunicação ética e empática na qual os atos de fala têm valor e reconhecimento entre todos os falantes ${ }^{14}$. Para isso, a relação de confiança entre o mentor e o grupo se mostra fundamental para a participação de todos. Nela, os alunos se sentem seguros para contar histórias pessoais, para trazer problemas difíceis e delicados, e para buscar na experiência de vida do mentor a possibilidade de construir um caminho para si mesmos.

No caso aqui relatado, a primeira questão que surgiu diante da impossibilidade de manter os encontros face a face foi sobre o quanto a transposição para o modelo remoto permitiria a intersubjetividade. A experiência revelou ser uma questão difícil que precisa ser encarada. Não bastasse o fato de o encontro se estabelecer com uma interposição tecnológica que subtrai a densidade sensorial do contato real, a atitude dos alunos de manter fechadas as câmeras, subtraindo também o contato imagético, prejudica a comunicação. A ausência da linguagem não verbal visual dificulta o reconhecimento de sentimentos, emoções e sinais que impactam a construção de vínculo e a transmissão e compreensão dos enunciados verbais.

Os motivos que levaram os alunos tão frequentemente a não abrir as câmeras é tema que extrapola os limites dessa experiência, mas que merece ser investigado, principalmente considerando que a interação remota tende a ser incorporada ao ensino pós-pandemia. Uma discreta pista que apareceu nessa experiência se refere à necessidade de estabelecer relações de confiança entre as pessoas quando do encontro remoto.
Ainda assim, os encontros de mentoria virtual foram avaliados como muito bons tanto por alunos quanto por mentores. Na mentoria dos calouros e dos veteranos, os alunos se sentiram cuidados, fortalecidos e gratos. Os mentores também compartilharam a percepção de satisfação com os encontros. Ou seja, houve uma boa interação entre as pessoas, produzindo efeitos benéficos perceptíveis.

Essas observações vêm ao encontro dos estudos ${ }^{11,13,15}$ que reconhecem a mentoria como um importante espaço de ressignificação das vivências estudantis, reforçando sua prescrição nos cursos médicos, com a ressalva de que tais estudos se deram em ambientes presenciais reais, enquanto, nessa experiência, tratamos do tema em ambiente remoto.

Esse resultado aponta para a necessidade de outros questionamentos sobre os modos de construção da subjetividade na pós-modernidade ${ }^{14}$, em um mundo midiático, tecnológico, subjetivista e ainda um tanto desconhecido para seus contemporâneos no que se refere aos efeitos de longo prazo na produção da pessoalidade, tema central nos programas de mentoria.

Outra questão que surgiu da experiência de mentoria virtual foi a dimensão ética do encontro. Em qualquer formato que se adote, os encontros de mentoria devem ser selados por acordos de sigilo e confidencialidade dos assuntos tratados em grupo. Na prática, esses acordos selam também a viabilidade do grupo, pois, sem segurança para falar, não se fala de assuntos que verdadeiramente interessam ao grupo.

$\mathrm{Na}$ modalidade remota, o acordo de sigilo e confidencialidade se manteve, mas cabe observar que o ambiente virtual é um ambiente de menor controle, pois pode ser gravado, filmado e depois manipulado sem que as pessoas envolvidas estejam cientes e de acordo. O mentor deverá estar atento e reforçar a discussão ética com seu grupo de alunos, buscando um compromisso mais forte e consciente por parte de todos sobre a responsabilidade de proteger os encontros e respeitar limites éticos, o que não deixa de ser um importante aprendizado para a vida pessoal e profissional.

A experiência da mentoria virtual em tempos de isolamento social em razão da pandemia de Sars-CoV-2 mostrou-se relevante e promissora. As questões aqui levantadas para reflexão, assim como o período relativamente curto da experiência, são limites deste estudo que devem ser considerados e apontam para a necessidade de outras e futuras investigações sobre o tema.

\section{AGRADECIMENTOS}

Agradecemos aos professores-mentores Cristiane Duez Verzaro dos Santos, Elaine Mendes de Oliveira Fernandes, Luiz Cláudio Lacerda, Allysson Coelho Sampaio e Bernardo 
Rahe o engajamento e a dedicação ao Programa de Mentoria Acadêmica do Curso de Medicina da Faculdade Santa Marcelina (FASM).

\section{CONTRIBUIÇÃO DOS AUTORES}

Izabel Cristina Rios realizou a concepção do estudo, planejou e coordenou o desenvolvimento do projeto tema da experiência relatada e elaborou o desenho do artigo, a revisão de literatura, o registro e a análise dos dados, a discussão dos resultados e a redação final do artigo. Edson Vanderlei Zombini participou do planejamento do estudo, desenvolveu atividades do projeto, contribuiu para o registro e a análise dos dados, participou da discussão dos resultados e colaborou na redação do texto. Martim Elviro de Medeiros Junior, Maria Teresa de Almeida Fernandes, Maira Kassabian Oliveira Pacheco, Elcio Gomes Mascarenhas e Joselita Batista Azuma participaram do planejamento do estudo, desenvolveram atividades do projeto, contribuíram para o registro e a análise dos dados, e colaboraram na discussão dos resultados e na revisão do texto. Pedro Felix Vital Junior participou da concepção, do planejamento e da implantação do projeto, da análise dos dados e da discussão dos resultados.

\section{CONFLITO DE INTERESSES}

Declaramos não haver conflito de interesses.

\section{FINANCIAMENTO}

Declaramos não haver financiamento.

\section{REFERÊNCIAS}

1. Sandhu P, Wolf M. The impact of Covid-19 on the undergraduate medical curriculum. Med Educ Online. 2020;25:1.

2. Kam SXL, Toledo ALS de, Pacheco CC, Souza GFB de, Santana VLM, BonfáAraujo $B$, et al. Estresse em estudantes ao longo da graduação médica. Rev Bras Educ Med. 2019;43(1 Supl 1):246-53.

3. Dyrbye LN, Shanafelt T. A narrative review on burnout experienced by medical students and residents. Med Educ. 2016;50(1):132-49.

4. Henderson M, Hope V. Medical student depression, anxiety and distress outside North America: a systematic review. Med Educ. 2014;48(10):963-79.

5. Dyrbye LN, West CP, Satele D, Boone S, Tan L, Sloan J, et al. Burnout among U.S. medical students, residents, and early career physicians relative to the general U.S. population. Acad Med. 2014;89(3):443-51.

6. Pacheco JP, Giacomin HT, Tam WW, Ribeiro TB, Arab C, Bezerra IM, et al. Mental health problems among medical students in Brazil: a systematic review and meta-analysis. J Bras Psiquiatr. 2017;39(4):369-78.

7. Maia BR, Dias PC. Ansiedade, depressão e estresse em estudantes universitários: o impacto da Covid-19. Estud Psicol (Campinas). 2020;37:e200067.

8. O'Byrne L, Gavin B, Adamis D, Lim YX, McNicholas F. Levels of stress in medical students due to Covid-19. J Med Ethics. 2021;0:1-6.

9. Teixeira LAC, Costa RA, Mattos RMPR, Pimentel D. Saúde mental dos estudantes de Medicina do Brasil durante a pandemia da coronavirus disease 2019. J Bras Psiquiatr. 2021; 70(1):21-9.

10. Sng JH, Pei Y, Toh HP, Peh TY, Neo SH, Krishna LKR. Mentoring relationships between senior physicians and junior doctors and/or medical students: a thematic review. Med Teach. 2017; 39:8.

11. Skjevik EP, Boudreau JD, Ringberg U, Schei E, Stenfors T, Kvernenes M, et al. Group mentorship for undergraduate medical students-a systematic review. Perspect Med Educ. 2020;9(5):272-80.

12. Martins AF, Bellodi PL. Mentoring: uma vivência de humanização e desenvolvimento no curso médico. Interface. 2016;20(58):715-26.

13. Silva JEC, Pontes RF, Costa JCT, Sobral JF. Mentoria para o estudante de medicina. Brazilian Journal of Development. 2021;7(4):42117-37.

14. Rios IC, Schraiber LB. Humanização e humanidades em medicina. São Paulo: Editora Unesp; 2012.

15. Bellodi PL, Martins MA. Tutoria: mentoring na formação médica. São Paulo: Casa do Psicólogo; 2005. 